\title{
GENERALIZED TELEGRAPH PROCESS WITH RANDOM JUMPS
}

\author{
ANTONIO DI CRESCENZO, ${ }^{* * *}$ \\ ANTONELLA IULIANO $* * * *$ AND \\ BARBARA MARTINUCCI, ${ }^{* * * * *}$ Università di Salerno \\ SHELEMYAHU ZACKS, ${ }^{* * * * *}$ Binghamton University
}

\begin{abstract}
We consider a generalized telegraph process which follows an alternating renewal process and is subject to random jumps. More specifically, consider a particle at the origin of the real line at time $t=0$. Then it goes along two alternating velocities with opposite directions, and performs a random jump toward the alternating direction at each velocity reversal. We develop the distribution of the location of the particle at an arbitrary fixed time $t$, and study this distribution under the assumption of exponentially distributed alternating random times. The cases of jumps having exponential distributions with constant rates and with linearly increasing rates are treated in detail.
\end{abstract}

Keywords: Jump-telegraph process; alternating renewal process; exponential random time; random jump

2010 Mathematics Subject Classification: Primary 60K15

Secondary $60 \mathrm{~J} 75$

\section{Introduction}

The telegraph process describes a random motion on the real line, where at each epoch of a Poisson process the speed of the running particle switches alternately between a positive (say $c$ ) and a negative $($ say $-v)$ preassigned velocity. Such a stochastic process has been investigated by several authors (see the initiatory contributions by Goldstein [11] and Kac [13]). Its probability density satisfies a hyperbolic differential equation, whose probabilistic properties were studied for instance by Orsingher [17] and Beghin et al. [1]. Moreover, the book of Pinsky [19] contains various results on the telegraph process, with its generalizations and applications. Some onedimensional generalizations of the telegraph process include the case of random velocities (see [24]), and the case of switching times governed by Erlang-distributed random variables (see [6]) or by an alternating renewal process (see [26]). The problem of the parametric estimation of the underlying rate of the Poisson process has been faced recently by De Gregorio and Iacus [4], [5].

The telegraph process deserves interest in various applied fields, such as actuarial and financial mathematics. For instance, we recall [16], where the relevance of the link between

\footnotetext{
Received 7 August 2012; revision received 15 September 2012.

* Postal address: Dipartimento di Matematica, Università di Salerno, Via Giovanni Paolo II, n. 132, Fisciano (SA) 84084, Italy.

** Email address: adicrescenzo@unisa.it

*** Email address: aiuliano@unisa.it

**** Email address: bmartinucci@ unisa.it

***** Postal address: Department of Mathematical Sciences, Binghamton University, Binghamton, NY 13902-6000, USA. Email address: shelly@math.binghamton.edu
} 
hitting times of risk processes and of wave governed random motions has been pinpointed. Moreover, a geometric telegraph process was proposed by Di Crescenzo and Pellerey [9] as a model to describe the dynamics of the price of risky assets. Aiming to refine such processes and looking for a model which is free of arbitrage and is complete, the jump-telegraph process has been recently introduced and studied by Ratanov [20], [21], and [22] and Ratanov and Melnikov [23]. This process includes the occurrence of a deterministic jump along the alternating direction at each velocity reversal. Other results on the jump-telegraph process, which include some limit theorems, are provided in [8] and [14]. Moreover, an application to option pricing models of the telegraph process with random jumps has appeared recently in [15].

According to the research stream described above, and following an approach based on investigating certain underlying compound renewal processes, in this paper we obtain the probability law of a generalized jump-telegraph process, say $X(t)$, subject to random jumps. In Section 2 we introduce in detail the model in its general form, and in particular define the random time $W(t)$, which describes the total time traveled by the particle with positive velocity up to time $t$. In Section 3 we express the distribution of $W(t)$ in terms of an auxiliary compound renewal process. This allows us to obtain, in Section 4, the distribution function of $X(t)$ in series form. Section 5 is devoted to investigating such distribution functions when the random times separating consecutive jumps are exponentially distributed. Two special cases are treated in detail, i.e. when the random jumps have exponential distribution (i) with constant rates, and (ii) with linearly increasing rates.

Finally, we remark that the main technique adopted in this paper, which is based on the examination of underlying compound renewal processes, has been suggested by previous investigations (see [18] for the analysis of compound Poisson processes with jumps), and has recently been successfully exploited by Bshouty et al. [3] for the study of a telegraph process with random delays. Furthermore, it should be noted that the results shown in the paper are also of interest to the analysis of growth-collapse models (see, for instance, [2]).

\section{The process}

Let $\{(X(t), V(t)) ; t \geq 0\}$ be a generalized jump-telegraph process on the real line, where, as usual, $X(t)$ and $V(t)$ respectively denote the position and velocity of the moving particle at time $t$. We assume that $X(0)=0$ and that, without loss of generality, the initial velocity is positive, i.e. $V(0)=c$. The motion thus goes upward for a random time $U_{1}$ at velocity $c$ $(c>0)$, and then goes downward for a random time $D_{1}$ at velocity $-v(v>0)$. Owing to the presence of random jumps, after the random time $U_{1}$ the process reverses the direction from upwards to downwards, but it jumps first upward a random height of length $J_{1}^{(\mathrm{U})}>0$, i.e.

$$
X\left(U_{1}^{-}\right)=c U_{1}, \quad X\left(U_{1}^{+}\right)=c U_{1}+J_{1}^{(\mathrm{U})} .
$$

The process then proceeds downwards for a random time $D_{1}$ and then makes a jump down of length $-J_{1}^{(\mathrm{D})}<0$, i.e.

$X\left(\left(U_{1}+D_{1}\right)^{-}\right)=c U_{1}-v D_{1}+J_{1}^{(\mathrm{U})}, \quad X\left(\left(U_{1}+D_{1}\right)^{+}\right)=c U_{1}-v D_{1}+J_{1}^{(\mathrm{U})}-J_{1}^{(\mathrm{D})}$.

This is one cycle of movements and jumps. After the first cycle a second cycle starts, and so on. All cycles are independent and follow the stochastic laws described below. More specifically, let $\left\{U_{1}, U_{2}, \ldots\right\}$ be a sequence of independent and identically distributed (i.i.d.) positive random variables, corresponding to movements upwards, having a common continuous distribution $F_{\mathrm{U}}(\cdot)$, with density $f_{\mathrm{U}}(\cdot)$. Similarly, let $\left\{D_{1}, D_{2}, \ldots\right\}$ be a sequence of i.i.d. positive random 


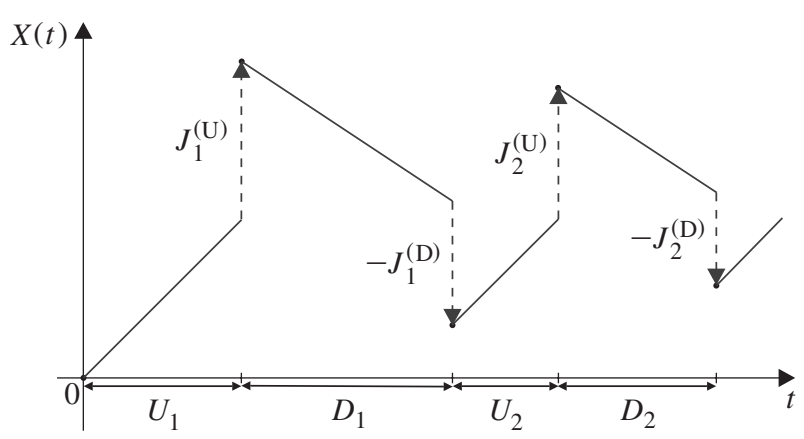

Figure 1: A sample path of $X(t)$.

variables for downwards movements, with a common distribution $F_{\mathrm{D}}(\cdot)$ having a density $f_{\mathrm{D}}(\cdot)$. As customary, for any distribution function $F(t)$, we set $\bar{F}(t)=1-F(t)$. For the jumps, let $\left\{J_{n}^{(\mathrm{U})}, n \geq 1\right\}$ and $\left\{J_{n}^{(\mathrm{D})}, n \geq 1\right\}$ be sequences of continuous positive independent random variables. We also assume that $\left\{U_{n}, n \geq 1\right\},\left\{D_{n}, n \geq 1\right\},\left\{J_{n}^{(\mathrm{U})}, n \geq 1\right\}$, and $\left\{J_{n}^{(\mathrm{D})}, n \geq 1\right\}$ are mutually independent. In Figure 1 we plot a realization of $\{X(t), t \geq 0\}$. We are interested in the distribution of $X(t)$, at a fixed time point, $0<t<+\infty$.

Let $W(t), 0<W(t) \leq t$, denote the total time in $(0, t]$ that the process $X(t)$ was moving up, i.e.

$$
W(t)=\int_{0}^{t} \mathbf{1}_{\{V(s)=c\}} \mathrm{d} s .
$$

The distribution of random time $W(t)$ is developed in the following section.

For $0<w<+\infty$, let us define the counting process

$$
N_{\mathrm{U}}(w)=\max \left\{n \geq 0: \sum_{i=1}^{n} U_{i} \leq w\right\}
$$

where, as customary, we assume that $\sum_{i=1}^{0} U_{i}=0$. Since every up movement is followed by an upward jump, the number of upward jumps in $(0, t]$ is $N_{\mathrm{U}}[W(t)]$. Moreover, since up movements and down movements alternate, the number of down jumps in $(0, t]$ is

$$
N_{\mathrm{D}}[W(t)]= \begin{cases}N_{\mathrm{U}}[W(t)] & \text { if } V(t)=c, \\ N_{\mathrm{U}}[W(t)]-1 & \text { if } V(t)=-v .\end{cases}
$$

Note that, due to (2), for $w>0$, we have

$$
\mathbb{P}\left\{N_{\mathrm{U}}(w)=n\right\}=F_{\mathrm{U}}^{(n)}(w)-F_{\mathrm{U}}^{(n+1)}(w), \quad n \geq 0,
$$

where $F_{\mathrm{U}}^{(n)}(w)$ is the distribution function of $U_{1}+\cdots+U_{n}, n \geq 1$, and $F_{\mathrm{U}}^{(0)}(w) \equiv 1, w \geq 0$. In the following, the $F_{\mathrm{D}}^{(n)}(w)$ will be defined similarly to $F_{\mathrm{U}}^{(n)}(w)$, and their density will be denoted as $f_{\mathrm{D}}^{(n)}(w)$ and $f_{\mathrm{U}}^{(n)}(w)$, respectively. Note that if $N_{\mathrm{U}}(w)=0$ then the moving particle is in the rising phase of its first cycle and, thus, $V(w)=c$. Thus, if $W(t)=w$ then

$$
X(t)=c w-v(t-w)+\sum_{n=1}^{N_{\mathrm{U}}(w)} J_{n}^{(\mathrm{U})}-\sum_{n=1}^{N_{\mathrm{D}}(w)} J_{n}^{(\mathrm{D})}, \quad t>0 .
$$

In the following section we investigate the probability law of the total time defined in (1), which will be useful to study the distribution of process $X(t)$ in Section 4. In the latter section the case of negative initial velocity will also be discussed. 


\section{The distribution of total time, $W(t)$}

We define the compound renewal process $\{Y(w), w \geq 0\}$, where

$$
Y(w)=\sum_{n=0}^{N_{\mathrm{U}}(w)} D_{n}, \quad w \geq 0
$$

with $D_{0} \equiv 0$. The cumulative distribution function of $Y(w)$ is

$$
F_{Y}(y ; w)=\mathbb{P}\{Y(w) \leq y\}=\sum_{n=0}^{+\infty} \mathbb{P}\left\{N_{\mathrm{U}}(w)=n\right\} F_{\mathrm{D}}^{(n)}(y), \quad y \geq 0 .
$$

Note that $F_{Y}\left(0^{-} ; w\right)=0, F_{Y}(0 ; w)=\bar{F}_{\mathrm{U}}(w)=1-F_{\mathrm{U}}(w)$, and, for $w \geq 0$, the probability density of process (6) is

$$
f_{Y}(y ; w)=\sum_{n=1}^{+\infty} \mathbb{P}\left\{N_{\mathrm{U}}(w)=n\right\} f_{\mathrm{D}}^{(n)}(y), \quad y>0 .
$$

We remark that the total time process (1) identifies with the stopping time

$$
W(t)=\inf \{w>0: Y(w) \geq t-w\}, \quad 0<w \leq t<+\infty .
$$

Furthermore, note that

(i) if $V(t)=c$ then $Y[W(t)]=t-W(t)$,

(ii) if $V(t)=-v$ then $Y[W(t)]>t-W(t)$.

Examples concerning cases (i) and (ii) are shown in Figure 2. In the following we express the absolutely continuous component and the discrete component of the probability law of $W(t)$.

Proposition 1. The probability law of $W(t)$ is expressed by

$$
\begin{aligned}
\mathbb{P}\{W(t)>w\} & =F_{Y}(t-w ; w) \\
& =\sum_{n=0}^{+\infty}\left[F_{\mathrm{U}}^{(n)}(w)-F_{\mathrm{U}}^{(n+1)}(w)\right] F_{\mathrm{D}}^{(n)}(t-w), \quad 0 \leq w<t,
\end{aligned}
$$

and by

$$
\mathbb{P}\{W(t)=t\}=\bar{F}_{\mathrm{U}}(t) .
$$

Proof. The proof easily follows from the relation between processes $W(t)$ and $Y(w)$, given by (9), from the increasingness of $Y(w)$, and from (4) and (7).

The probability law of $W(t)$ has an absolutely continuous component, whose density will be denoted $f_{W}(w ; t)$. It will be expressed in terms of the following subdensities:

$$
\begin{aligned}
\psi_{c}(w, t) & :=\frac{\mathrm{d}}{\mathrm{d} w} \mathbb{P}\{W(t) \leq w, V(t)=c\}, \\
\psi_{-v}(w, t) & :=\frac{\mathrm{d}}{\mathrm{d} w} \mathbb{P}\{W(t) \leq w, V(t)=-v\} .
\end{aligned}
$$



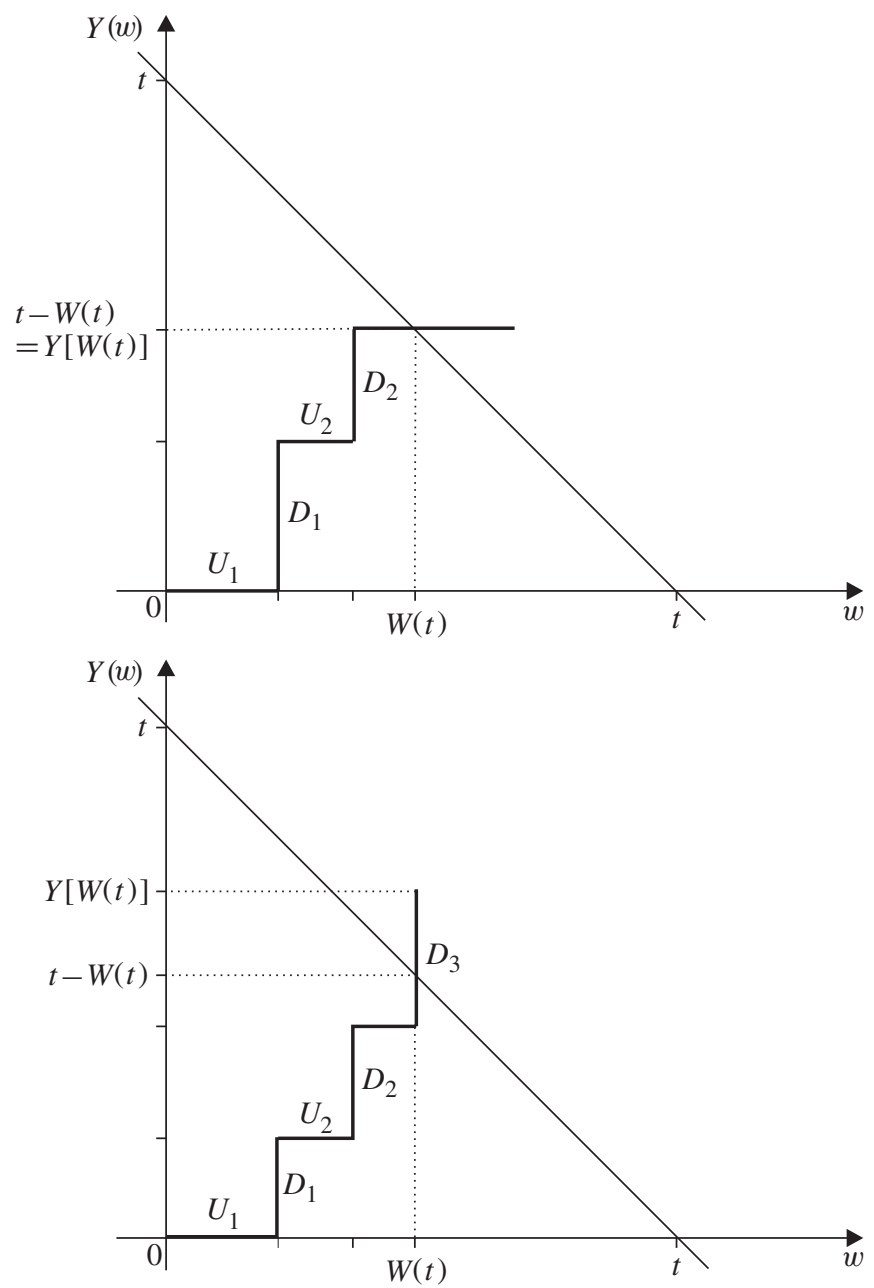

FIGURE 2: Sample paths of $Y(w)$ and $W(t)$, where $V(t)=c$ (top) and $V(t)=-v$ (bottom).

Corollary 1. The density of $W(t)$ is given by

$$
f_{W}(w ; t)=\psi_{c}(w, t)+\psi_{-v}(w, t), \quad 0<w<t,
$$

where

$$
\psi_{c}(w, t)=f_{Y}(t-w, w)=\sum_{n=1}^{+\infty}\left[F_{\mathrm{U}}^{(n)}(w)-F_{\mathrm{U}}^{(n+1)}(w)\right] f_{\mathrm{D}}^{(n)}(t-w)
$$

and

$$
\psi_{-v}(w, t)=\sum_{n=0}^{+\infty}\left[F_{\mathrm{D}}^{(n)}(t-w)-F_{\mathrm{D}}^{(n+1)}(t-w)\right] f_{\mathrm{U}}^{(n+1)}(w) .
$$

Proof. If $V(t)=c$, which corresponds to the top diagram of Figure 2, then $W(t)=w$ if and only if $Y(w)=t-w$. Hence, the subdensity $\psi_{c}(w, t)$ is equal to $f_{Y}(t-w, w)$. This gives the first identity of (12), whereas the second identity immediately follows from (4) and (8). Equation (13) is obtained by a similar reasoning and recalling that $V(0)=c$. The proof of (11) follows by differentiation of (10), and by rearranging the terms. 


\section{The distribution of particle position}

In order to determine the distribution function of $X(t)$, we give some necessary notions.

The jumps random variables $\left\{\left(J_{n}^{(\mathrm{U})}, J_{n}^{(\mathrm{D})}\right), n \geq 1\right\}$ are independent of $\left\{U_{n}, D_{n}, n \geq 1\right\}$. Therefore, $\left(J_{n}^{(\mathrm{U})}, J_{n}^{(\mathrm{D})}\right)$ are conditionally independent of $\left\{N_{\mathrm{U}}(w), w>0\right\}$, given $\left\{N_{\mathrm{U}}(w)=\right.$ $n$ \}. Let us define the net displacement performed by the effect of jumps at the $n$th cycle:

$$
Z_{n}=J_{n}^{(\mathrm{U})}-J_{n}^{(\mathrm{D})}, \quad n \geq 1 .
$$

Note that the random variables $Z_{n}, n \geq 1$, are independent, but not necessarily identically distributed. Let us now introduce the distribution functions

$$
A^{(n)}(x)=\mathbb{P}\left\{J_{1}^{(\mathrm{U})}+\cdots+J_{n}^{(\mathrm{U})} \leq x\right\}, \quad B^{(n)}(x)=\mathbb{P}\left\{J_{1}^{(\mathrm{D})}+\cdots+J_{n}^{(\mathrm{D})} \leq x\right\},
$$

for $x \in \mathbb{R}$, and let $a^{(n)}(x)$ and $b^{(n)}(x)$ be the corresponding probability densities. Moreover, for $z \in \mathbb{R}$, we define the two distribution functions

$$
K^{(n)}(z)=\mathbb{P}\left\{Z_{1}+\cdots+Z_{n} \leq z\right\}=\int_{\max (-z, 0)}^{\infty} A^{(n)}(z+y) b^{(n)}(y) \mathrm{d} y
$$

and

$$
\tilde{K}^{(n)}(z)=\mathbb{P}\left\{Z_{1}+\cdots+Z_{n-1}+J_{n}^{(\mathrm{U})} \leq z\right\}=\int_{\max (-z, 0)}^{\infty} A^{(n)}(z+y) b^{(n-1)}(y) \mathrm{d} y .
$$

They are involved in the probability law of $X(t)$, as shown in the following theorem.

Theorem 1. The distribution function of $X(t)$ is, for $t>0$ and $\xi \in \mathbb{R}$,

$$
\begin{gathered}
\mathbb{P}\{X(t) \leq \xi\}=\bar{F}_{\mathrm{U}}(t) \mathbf{1}_{\{\xi \geq c t\}} \\
+\sum_{n=1}^{+\infty} \int_{0}^{t}\left\{K^{(n)}(\xi+v t-w(c+v))\left[F_{\mathrm{U}}^{(n)}(w)-F_{\mathrm{U}}^{(n+1)}(w)\right] f_{\mathrm{D}}^{(n)}(t-w)\right. \\
+\tilde{K}^{(n)}(\xi+v t-w(c+v)) \\
\left.\quad \times\left[F_{\mathrm{D}}^{(n-1)}(t-w)-F_{\mathrm{D}}^{(n)}(t-w)\right] f_{\mathrm{U}}^{(n)}(w)\right\} \mathrm{d} w
\end{gathered}
$$

Proof. Since $\mathbb{P}\{X(t) \leq \xi \mid W(t)=t\}=\mathbf{1}_{\{c t \leq \xi\}}$, and making use of (11), the cumulative distribution function of $X(t)$ can be expressed as

$$
\begin{aligned}
\mathbb{P}\{X(t) \leq \xi\}= & \bar{F}_{\mathrm{U}}(t) \mathbf{1}_{\{c t \leq \xi\}}+\int_{0}^{t} \mathbb{P}\{X(t) \leq \xi \mid W(t)=w\} \psi_{c}(w ; t) \mathrm{d} w \\
& +\int_{0}^{t} \mathbb{P}\{X(t) \leq \xi \mid W(t)=w\} \psi_{-v}(w ; t) \mathrm{d} w
\end{aligned}
$$

Recalling (15) and (16), owing to (5), we have

$$
\mathbb{P}\left\{X(t) \leq \xi \mid W(t)=w, N_{\mathrm{U}}(w)=n, V(t)=c\right\}=K^{(n)}(\xi+v t-w(c+v))
$$

and

$$
\mathbb{P}\left\{X(t) \leq \xi \mid W(t)=w, N_{\mathrm{U}}(w)=n, V(t)=-v\right\}=\tilde{K}^{(n)}(\xi+v t-w(c+v)) .
$$


Moreover, it is not hard to see that, for $n \geq 1$, we have

$$
\mathbb{P}\left\{N_{\mathrm{U}}(w)=n \mid W(t)=w, V(t)=c\right\}=\frac{\left[F_{\mathrm{U}}^{(n)}(w)-F_{\mathrm{U}}^{(n+1)}(w)\right] f_{\mathrm{D}}^{(n)}(t-w)}{\psi_{c}(w ; t)}
$$

and

$$
\mathbb{P}\left\{N_{\mathrm{U}}(w)=n \mid W(t)=w, V(t)=-v\right\}=\frac{\left[F_{\mathrm{D}}^{(n-1)}(t-w)-F_{\mathrm{D}}^{(n)}(t-w)\right] f_{\mathrm{U}}^{(n)}(w)}{\psi_{-v}(w ; t)} .
$$

Hence, from (18)-(21), it follows that

$$
\begin{aligned}
& \mathbb{P}\{X(t) \leq \xi \mid W(t)=w, V(t)=c\} \\
& \quad=\frac{1}{\psi_{c}(w ; t)} \sum_{n=1}^{+\infty} K^{(n)}(\xi+v t-w(c+v))\left[F_{\mathrm{U}}^{(n)}(w)-F_{\mathrm{U}}^{(n+1)}(w)\right] f_{\mathrm{D}}^{(n)}(t-w)
\end{aligned}
$$

and

$$
\begin{aligned}
& \mathbb{P}\{X(t) \leq \xi \mid W(t)=w, V(t)=-v\} \\
& \quad=\frac{1}{\psi_{-v}(w ; t)} \sum_{n=1}^{+\infty} \tilde{K}^{(n)}(\xi+v t-w(c+v))\left[F_{\mathrm{D}}^{(n-1)}(t-w)-F_{\mathrm{D}}^{(n)}(t-w)\right] f_{\mathrm{U}}^{(n)}(w) .
\end{aligned}
$$

We note that the conditional distribution of $V(t)$, given $W(t)$, is

$$
\mathbb{P}\{V(t)=c \mid W(t)=w\}=\frac{\psi_{c}(w ; t)}{f_{W}(w ; t)}, \quad \mathbb{P}\{V(t)=-v \mid W(t)=w\}=\frac{\psi_{-v}(w ; t)}{f_{W}(w ; t)},
$$

so that from (22) and (23) we obtain

$$
\begin{aligned}
& \mathbb{P}\{X(t) \leq \xi \mid W(t)=w\} \\
& =\frac{1}{f_{W}(w ; t)} \sum_{n=1}^{+\infty}\left\{K^{(n)}(\xi+v t-w(c+v))\left[F_{\mathrm{U}}^{(n)}(w)-F_{\mathrm{U}}^{(n+1)}(w)\right] f_{\mathrm{D}}^{(n)}(t-w)\right. \\
& \left.\quad \quad+\tilde{K}^{(n)}(\xi+v t-w(c+v))\left[F_{\mathrm{D}}^{(n-1)}(t-w)-F_{\mathrm{D}}^{(n)}(t-w)\right] f_{\mathrm{U}}^{(n)}(w)\right\} .
\end{aligned}
$$

Distribution (17) thus finally follows.

The remaining part of this section is devoted to the analysis of the random motion when the initial velocity is negative. Let us denote by $X^{*}(t), t \geq 0$, the position of the particle at time $t$ when $V(0)=-v$. In this case the motion goes downward for a random time $D_{1}$ at velocity $-v$, then an instantaneous downward jump of random length $-J_{1}^{(\mathrm{D})}<0$ occurs. The motion thus goes upward for a random time $U_{1}$ at velocity $c$, and an instantaneous upward jump of random length $J_{1}^{(\mathrm{U})}>0$ finally occurs. This concludes the first cycle of movements and jumps. The second cycle then starts and the motion proceeds so on. In this case, similarly to (3) we define

$$
N_{\mathrm{D}}^{*}[W(t)]= \begin{cases}N_{\mathrm{U}}[W(t)] & \text { if } V(t)=-v, \\ N_{\mathrm{U}}[W(t)]+1 & \text { if } V(t)=c .\end{cases}
$$

Hence, when the initial velocity is negative, if $W(t)=w$ then the particle position at time $t$ can be expressed as

$$
X^{*}(t)=c w-v(t-w)+\sum_{n=1}^{N_{\mathrm{U}}(w)} J_{n}^{(\mathrm{U})}-\sum_{n=1}^{N_{\mathrm{D}}^{*}(w)} J_{n}^{(\mathrm{D})}, \quad t>0
$$


in analogy with (5). The probability law of (24) is thus similar to that of $X(t)$, due to the symmetry of the sample paths of the two processes. Indeed, the following result holds in analogy with Theorem 1, and therefore the proof is omitted.

Theorem 2. The distribution function of $X^{*}(t)$ is, for $t>0$ and $\xi \in \mathbb{R}$,

$$
\begin{aligned}
& \mathbb{P}\left\{X^{*}(t) \leq \xi\right\}= \bar{F}_{\mathrm{D}}(t) \mathbf{1}_{\{\xi \leq-v t\}} \\
&+\sum_{n=1}^{+\infty} \int_{0}^{t}\left\{K^{(n)}(-\xi+c t-w(c+v))\left[F_{\mathrm{D}}^{(n)}(w)-F_{\mathrm{D}}^{(n+1)}(w)\right] f_{\mathrm{U}}^{(n)}(t-w)\right. \\
& \quad+\tilde{K}^{(n)}(-\xi+c t-w(c+v)) \\
&\left.\quad \times\left[F_{\mathrm{U}}^{(n-1)}(t-w)-F_{\mathrm{U}}^{(n)}(t-w)\right] f_{\mathrm{D}}^{(n)}(w)\right\} \mathrm{d} w .
\end{aligned}
$$

In the following section we consider some special cases.

\section{Exponential times}

Let us now assume that the random times separating consecutive jumps (and velocity changes) have exponential distribution. Specifically, for all $n=1,2, \ldots$, let $U_{n}$ and $D_{n}$ be exponentially distributed with parameters $\eta$ and $\theta$, respectively, i.e.

$$
F_{\mathrm{U}}(x)=1-\mathrm{e}^{-\eta x}, \quad F_{\mathrm{D}}(x)=1-\mathrm{e}^{-\theta x}, \quad x \geq 0 .
$$

In the following, for a Poisson distribution with mean $\zeta$, the probability mass function and the cumulative distribution function will be respectively denoted as

$$
p(n, \zeta)=\frac{\zeta^{n}}{n !} \mathrm{e}^{-\zeta}, \quad P(n, \zeta)=\mathrm{e}^{-\zeta} \sum_{i=0}^{n} \frac{\zeta^{i}}{i !}, \quad n=0,1,2, \ldots
$$

Hence, we have $F_{\mathrm{U}}^{(n)}(w)=1-P(n-1, \eta w), w>0$. Lemma 1 below thus follows.

Lemma 1. For $w \geq 0$ and $n=1,2, \ldots$, the assumptions in (25) yield

$$
\left[F_{\mathrm{U}}^{(n)}(w)-F_{\mathrm{U}}^{(n+1)}(w)\right] f_{\mathrm{D}}^{(n)}(t-w)=\theta p(n, \eta w) p(n-1, \theta(t-w))
$$

and

$$
\left[F_{\mathrm{D}}^{(n-1)}(t-w)-F_{\mathrm{D}}^{(n)}(t-w)\right] f_{\mathrm{U}}^{(n)}(w)=\eta p(n-1, \eta w) p(n-1, \theta(t-w)),
$$

with the function $p(\cdot, \cdot)$ defined in (26).

\subsection{Exponential times and exponential jumps}

We consider the case in which the jump lengths $J_{k}^{(\mathrm{U})}$ (and $J_{k}^{(\mathrm{D})}$ ) are exponentially distributed and i.i.d. for $k=1,2, \ldots$. Hence, assume that

$$
\mathbb{P}\left\{J_{k}^{(\mathrm{U})} \leq x\right\}=1-\mathrm{e}^{-\lambda x}, \quad \mathbb{P}\left\{J_{k}^{(\mathrm{D})} \leq x\right\}=1-\mathrm{e}^{-\mu x}, \quad x \geq 0 .
$$

We recall that, by the Poisson-gamma relationship, for $n=1,2, \ldots$, from (14) we have the Erlang distribution

$$
A^{(n)}(x)=1-P(n-1 ; \lambda x)=1-\mathrm{e}^{-\lambda x} \sum_{i=0}^{n-1} \frac{(\lambda x)^{i}}{i !}, \quad x \geq 0,
$$


where $P(\cdot, \cdot)$ is defined in (26). The densities corresponding to $A^{(n)}(x)$ and $B^{(n)}(x)$ are thus

$$
a^{(n)}(x)=\frac{\lambda^{n} x^{n-1}}{(n-1) !} \mathrm{e}^{-\lambda x}, \quad b^{(n)}(x)=\frac{\mu^{n} x^{n-1}}{(n-1) !} \mathrm{e}^{-\mu x}, \quad x \geq 0 .
$$

By using (17), the following theorem gives explicit formulae for $K^{(n)}(z)$ and $\tilde{K}^{(n)}(z)$.

Theorem 3. Let the random times and the jumps be exponentially distributed as respectively specified in (25) and (27). Then, for $n \geq 1$ and $z \in \mathbb{R}$,

$$
\begin{aligned}
K^{(n)}(z)=\mathbf{1}_{\{z \geq 0\}}\{1 & \left.-\left(\frac{\mu}{\lambda+\mu}\right)^{n} \sum_{k=0}^{n-1}\left(\begin{array}{c}
n+k-1 \\
k
\end{array}\right)\left(\frac{\lambda}{\lambda+\mu}\right)^{k} P(n-k-1 ; \lambda z)\right\} \\
+\mathbf{1}_{\{z<0\}}\{ & P(n-1 ; \mu|z|) \\
& -\left(\frac{\mu}{\lambda+\mu}\right) \sum_{k=0}^{n-1}\left(\begin{array}{c}
n+k-1 \\
k
\end{array}\right)\left(\frac{\lambda}{\lambda+\mu}\right)^{k} P(n+k-1,(\lambda+\mu)|z|) \\
& \left.\times \sum_{l=0}^{n-k-1} \mathrm{e}^{-\lambda z} \frac{(\lambda z)^{l}}{l !}\right\}
\end{aligned}
$$

Similarly, for $n \geq 1$ and $z \in \mathbb{R}$,

$$
\begin{aligned}
\tilde{K}^{(n)}(z)=\mathbf{1}_{\{z \geq 0\}}\{1- & \left.\left(\frac{\mu}{\lambda+\mu}\right)^{n-1} \sum_{k=0}^{n-1}\left(\begin{array}{c}
n+k-2 \\
k
\end{array}\right)\left(\frac{\lambda}{\lambda+\mu}\right)^{k} P(n-k-1 ; \lambda z)\right\} \\
+\mathbf{1}_{\{z<0, n \geq 2\}}\{ & P(n-2 ; \mu|z|) \\
& -\left(\frac{\mu}{\lambda+\mu}\right)^{n-1} \sum_{k=0}^{n-1}\left(\begin{array}{c}
n+k-2 \\
k
\end{array}\right)\left(\frac{\lambda}{\lambda+\mu}\right)^{k} \\
\times & \left.P(n+k-2 ;(\lambda+\mu)|z|) \sum_{l=0}^{n-k-1} \mathrm{e}^{-\lambda z} \frac{(\lambda z)^{l}}{l !}\right\} .
\end{aligned}
$$

Proof. For $z \geq 0$, according to (15), we have

$$
\begin{aligned}
K^{(n)}(z) & =\int_{0}^{\infty}\left(1-\mathrm{e}^{-\lambda(z+y)} \sum_{i=0}^{n-1} \frac{\lambda^{i}(z+y)^{i}}{i !}\right)\left(\frac{\mu^{n} y^{n-1}}{(n-1) !} \mathrm{e}^{-\mu y}\right) \mathrm{d} y \\
& =1-\mathrm{e}^{\lambda z} \frac{\mu^{n}}{(n-1) !} \sum_{i=0}^{n-1} \frac{\lambda^{i}}{i !} \sum_{k=0}^{i}\left(\begin{array}{l}
i \\
k
\end{array}\right) z^{i-k} \int_{0}^{\infty} y^{n+k-1} \mathrm{e}^{-(\mu+\lambda) y} \mathrm{~d} y \\
& =1-\left(\frac{\mu}{\lambda+\mu}\right)^{n} \sum_{k=0}^{n-1}\left(\begin{array}{c}
n+k-1 \\
k
\end{array}\right)\left(\frac{\lambda}{\lambda+\mu}\right)^{k} P(n-k-1 ; \lambda z),
\end{aligned}
$$

whereas, if $z<0$,

$$
K^{(n)}(z)=\int_{|z|}^{\infty}[1-P(n-1 ; \lambda(z+y))] \frac{\mu^{n} y^{n-1}}{(n-1) !} \mathrm{e}^{-\mu y} \mathrm{~d} y .
$$


By some manipulations similar to those in (30), for $z<0$, we have

$$
\begin{aligned}
K^{(n)}(z)=P(n-1 ; \mu|z|)-\left(\frac{\mu}{\lambda+\mu}\right)^{n} & \sum_{k=0}^{n-1}\left(\begin{array}{c}
n+k-1 \\
k
\end{array}\right)\left(\frac{\lambda}{\lambda+\mu}\right)^{k} \\
& \times P(n+k-1,(\lambda+\mu)|z|) \sum_{l=0}^{n-k-1} \mathrm{e}^{-\lambda z \frac{(\lambda z)^{l}}{l !}}
\end{aligned}
$$

From (31) and (32), we thus obtain (28). Finally, (29) can be derived in a similar manner.

The functions $K^{(n)}(z)$ and $\tilde{K}^{(n)}(z)$ evaluated in Theorem 3 are essential to obtaining the distribution function of $X(t)$ via Theorem 1. However, an explicit form for $\mathbb{P}\{X(t) \leq \xi\}$ is very hard to obtain. Hence, we are forced to adopt a computational procedure finalized to its numerical evaluation. This is performed by substituting $z=\xi+v t-w(c+v)$ in (28) and (29), and taking into account the relations given in Lemma 1. Use of such quantities is thus made in (17) to numerically evaluate the distribution function of $X(t)$. We omit the computational details and present in Figure 3 some plots of the corresponding probability density function $f_{X_{t}}(\xi):=\mathrm{d} \mathbb{P}\{X(t) \leq \xi\} / \mathrm{d} t$. They show that the density spreads out when $t$ increases, which is in agreement with the behavior of the probability densities of similar processes studied in the past (see, for instance, the telegraph process with constant jumps studied in [8]). Moreover, a straightforward comparison shows that, for fixed $t$, the densities obtained in the case of random jumps are smoother than those obtained when the jumps are constant. In addition,
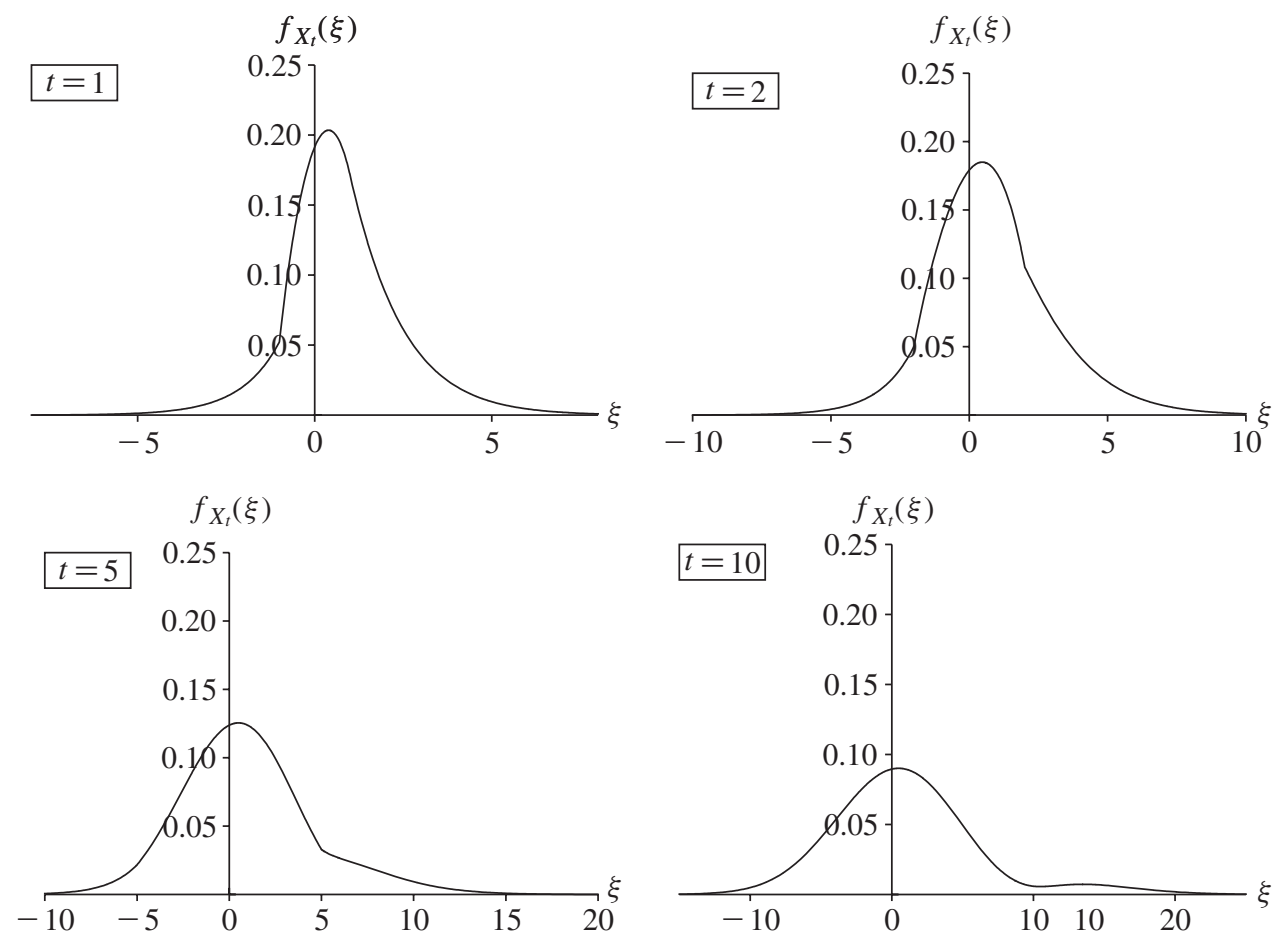

Figure 3: Density function of $X(t)$ for $\lambda=\mu=1, \eta=\theta=1, c=v=1$, and some choices of $t$ for exponential times and exponential jumps treated in Section 5.1. 
Figure 3 confirms that the density $f_{X_{t}}(\xi)$ has three different expressions in the intervals $\xi \leq-v t$, $-v t<\xi<c t$, and $\xi \geq c t$, depending on the form of $K^{(n)}(\cdot)$ and $\tilde{K}^{(n)}(\cdot)$ in (17).

\subsection{Exponential times and exponential jumps with linear rates}

Stimulated by the examples of the stochastic models treated in [7] and [10], in this section we study the case in which the jump lengths are exponentially distributed with linearly increasing rates. Specifically, we assume that the random variables $J_{k}^{(\mathrm{U})}$ and $J_{k}^{(\mathrm{D})}, k=1,2, \ldots$, have exponential distribution with linear rates $\lambda k$ and $\mu k$, respectively, so that

$$
\mathbb{P}\left\{J_{k}^{(\mathrm{U})} \leq x\right\}=1-\mathrm{e}^{-\lambda k x}, \quad \mathbb{P}\left\{J_{k}^{(\mathrm{D})} \leq x\right\}=1-\mathrm{e}^{-\mu k x}, \quad x \geq 0,
$$

with $\lambda, \mu>0$. From (14) we have, for $n=1,2, \ldots$,

$$
A^{(n)}(x)=\left(1-\mathrm{e}^{-\lambda x}\right)^{n}, \quad B^{(n)}(x)=\left(1-\mathrm{e}^{-\mu x}\right)^{n}, \quad x \geq 0 .
$$

The corresponding densities are thus

$$
a^{(n)}(x)=n\left(1-\mathrm{e}^{-\lambda x}\right)^{n-1} \lambda \mathrm{e}^{-\lambda x}, \quad b^{(n)}(x)=n\left(1-\mathrm{e}^{-\mu x}\right)^{n-1} \mu \mathrm{e}^{-\mu x}, \quad x \geq 0 .
$$

This can be seen, for instance, from Lemma 1 of [25]. Hypothesis (33) implies that the mean jump sizes $\mathbb{E}\left[J_{k}^{(\mathrm{U})}\right]=(\lambda k)^{-1}$ and $\mathbb{E}\left[J_{k}^{(\mathrm{D})}\right]=(\mu k)^{-1}$ decrease when $k$ increases, since $J_{k}^{(\mathrm{U})}$ and $J_{k}^{(\mathrm{D})}$ are stochastically decreasing with $k$. It follows that the sequence of jump sizes exhibits a damped character.

The following theorem gives explicit formulae for $K^{(n)}(z)$ and $\tilde{K}^{(n)}(z)$.

Theorem 4. Let the jumps be exponentially distributed with linear rates as specified in (33), with $\lambda>0$ and $\mu>0$. Then, for $n \geq 1$ and $z \in \mathbb{R}$,

$$
\begin{aligned}
K^{(n)}(z)= & \mathbf{1}_{\{z \geq 0\}} n \sum_{k=0}^{n}\left(\begin{array}{l}
n \\
k
\end{array}\right)\left(-\mathrm{e}^{-\lambda z}\right)^{k} B\left(1+k \frac{\lambda}{\mu}, n\right) \\
& +\mathbf{1}_{\{z<0\}} n \sum_{k=0}^{n}\left(\begin{array}{l}
n \\
k
\end{array}\right)\left(-\mathrm{e}^{-\lambda z}\right)^{k} B\left(\mathrm{e}^{\mu z} ; 1+k \frac{\lambda}{\mu}, n\right)
\end{aligned}
$$

and

$$
\begin{aligned}
\tilde{K}^{(n)}(z)= & \mathbf{1}_{\{z \geq 0\}}(n-1) \sum_{k=0}^{n}\left(\begin{array}{l}
n \\
k
\end{array}\right)\left(-\mathrm{e}^{-\lambda z}\right)^{k} B\left(1+k \frac{\lambda}{\mu}, n-1\right) \\
& +\mathbf{1}_{\{z<0\}}(n-1) \sum_{k=0}^{n}\left(\begin{array}{l}
n \\
k
\end{array}\right)\left(-\mathrm{e}^{-\lambda z}\right)^{k} B\left(\mathrm{e}^{\mu z} ; 1+k \frac{\lambda}{\mu}, n-1\right),
\end{aligned}
$$

where $B(a, b)$ is the beta function and $B(x ; a, b)$ is the incomplete beta function.

Proof. Recalling (15)-(16) and (34)-(35), the proofs of (36) and (37) immediately follow upon noting that, for any $a \geq 0$,

$$
\begin{aligned}
\int_{a}^{+\infty} & \left(1-\mathrm{e}^{-\lambda(z+y)}\right)^{n} n\left(1-\mathrm{e}^{-\mu y}\right)^{n-1} \mu \mathrm{e}^{-\mu y} \mathrm{~d} y \\
= & n \sum_{k=0}^{n}\left(\begin{array}{l}
n \\
k
\end{array}\right)\left(-\mathrm{e}^{-\lambda z}\right)^{k} \int_{0}^{\mathrm{e}^{-\mu a}} x^{k \lambda / \mu}(1-x)^{n-1} \mathrm{~d} x,
\end{aligned}
$$

and making use of the definition of the beta and incomplete beta functions. 
Remark 1. If $\mu=\lambda$ then (36) and (37) can be rewritten in the following alternative way. For $n \geq 1$,

$$
\begin{gathered}
K^{(n)}(z)=\mathbf{1}_{\{z \geq 0\} 2} F_{1}\left(-n, 1 ; n+1 ; \mathrm{e}^{-\lambda z}\right)+\mathbf{1}_{\{z<0\}} \frac{n}{n+1} \mathrm{e}^{\lambda z} F_{1}\left(-n+1,1 ; n+2 ; \mathrm{e}^{\lambda z}\right), \\
\tilde{K}^{(n)}(z)=\mathbf{1}_{\{z \geq 0\} 2} F_{1}\left(-n, 1 ; n ; \mathrm{e}^{-\lambda z}\right)+\mathbf{1}_{\{z<0\}} \frac{n-1}{n+1} \mathrm{e}^{\lambda z}{ }_{2} F_{1}\left(-n+2,1 ; n+2 ; \mathrm{e}^{\lambda z}\right),
\end{gathered}
$$

where

$$
{ }_{2} F_{1}(a, b ; c ; z)=\sum_{n=0}^{+\infty} \frac{(a)_{n}(b)_{n}}{(c)_{n}} \frac{z^{n}}{n !}
$$

is the hypergeometric function, with $(a)_{0}=1$ and $(a)_{n}=a(a+1) \cdots(a+n-1)$ for $n \geq 1$ (the rising factorial). In this case, the expressions of $K^{(n)}(z)$ and $\tilde{K}^{(n)}(z)$ follow from $(15)$, (34), and (35), and making use of Equation 3.197.3 (when $z \geq 0$ ) and Equation 3.197.8 (when $z<0)$ of [12].

Resorting to Theorem 1 and Lemma 1 the distribution function of $X(t)$ could be analytically evaluated. However, its expression is omitted, being very cumbersome. We only mention that, under the assumptions of Theorem 4, it can be shown explicitly that the distribution function of $X(t)$ has different expressions in the following domains: $\xi \leq-v t,-v t<\xi<c t, \xi \geq c t$.

In conclusion, Figure 4 shows some plots of the density $f_{X_{t}}(\xi)$ for various choices of $t$. We note that the behavior of such a density is similar to, but more peaked than the density given
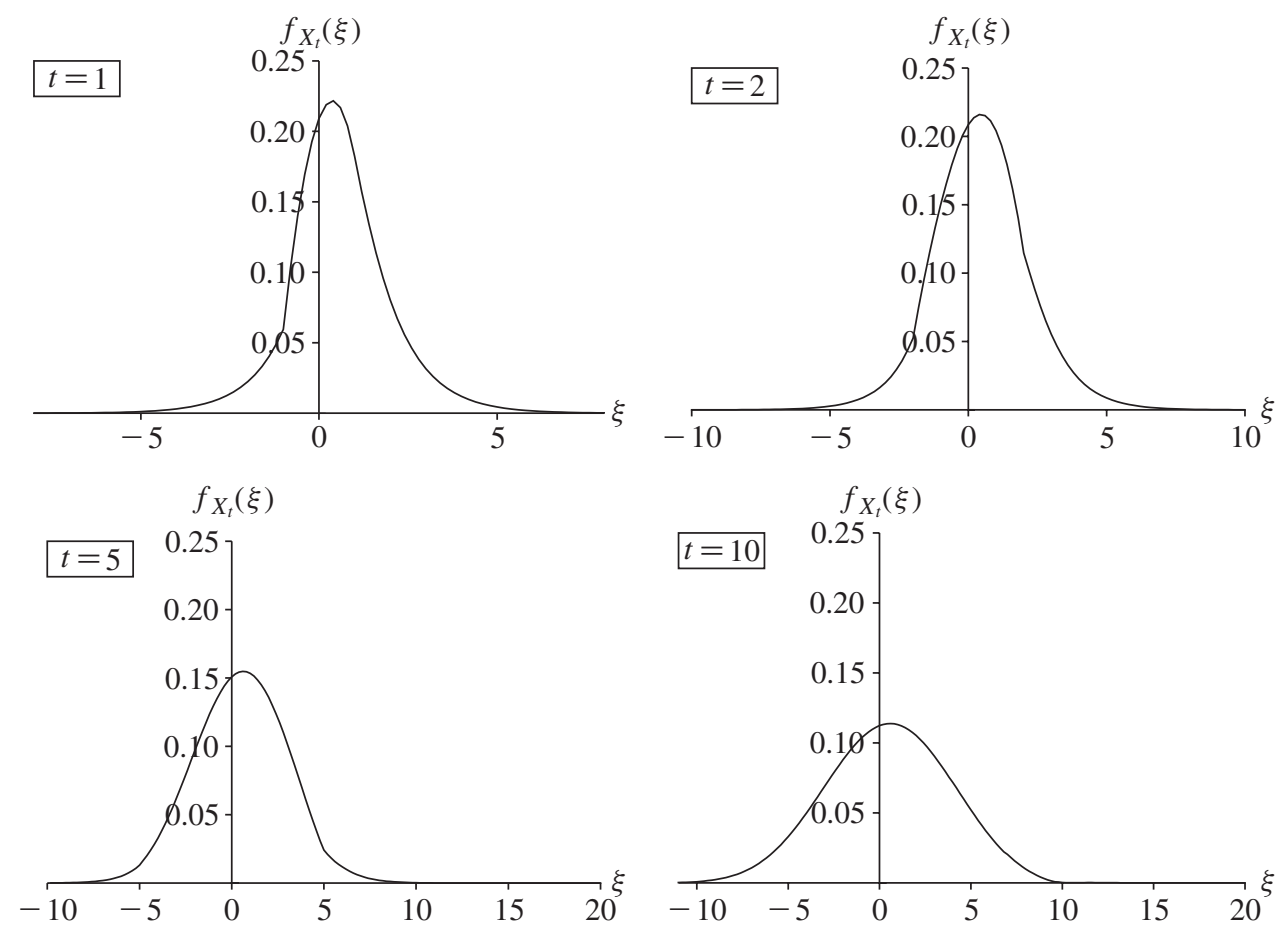

Figure 4: Density function of $X(t)$ for $\lambda=\mu=1, \eta=\theta=1, c=v=1$, and some choices of $t$ for exponential times and exponential jumps with linear rates treated in Section 5.2. 
in Figure 3. This is a consequence of the involved jump distributions (27) and (33). Indeed, for fixed values of $\lambda$ and $\mu$, in this case the jumps are stochastically smaller than those of Figure 3 .

\section{Acknowledgements}

The research of A. Di Crescenzo, A. Iuliano, and B. Martinucci was partially supported by the MIUR-PRIN 2008 'Mathematical models and computation methods for information processing and transmission in neuronal systems subject to stochastic dynamics'. The authors thank the anonymous referee for helpful comments that improved the paper.

\section{References}

[1] Beghin, L., Nieddu, L. And Orsingher, E. (2001). Probabilistic analysis of the telegrapher's process with drift by means of relativistic transformations. J. Appl. Math. Stoch. Anal. 14, 11-25.

[2] Boxma, O., Perry, D., Stadje, W. and Zacks, S. (2006). A Markovian growth-collapse model. Adv. Appl. Prob. 38, 221-243.

[3] Bshouty, D., Di Crescenzo, A., Martinucci, B. and Zacks, S. (2012). Generalized telegraph process with random delays. J. Appl. Prob. 49, 850-865.

[4] De Gregorio, A. and Iacus, S. M. (2008). Parametric estimation for standard and geometric telegraph process observed at discrete times. Statist. Infer. Stoch. Process. 11, 249-263.

[5] De Gregorio, A. and Iacus, S. M. (2011). Least-squares change-point estimation for the telegraph process observed at discrete times. Statistics 45, 349-359.

[6] Di Crescenzo, A. (2001). On random motions with velocities alternating at Erlang-distributed random times. Adv. Appl. Prob. 33, 690-701.

[7] Di Crescenzo, A. And Martinucci, B. (2010). A damped telegraph random process with logistic stationary distribution. J. Appl. Prob. 47, 84-96.

[8] Di Crescenzo, A. And Martinucci, B. (2013). On the generalized telegraph process with deterministic jumps. Methodology Comput. Appl. Prob. 15, 215-235.

[9] Di Crescenzo, A. And Pellerey, F. (2002). On prices' evolutions based on geometric telegrapher's process. Appl. Stoch. Models Business Industry 18, 171-184.

[10] Di Crescenzo, A., Martinucci, B. And Zacks, S. (2012). On the damped geometric telegrapher's process. In Mathematical and Statistical Methods for Actuarial Sciences and Finance, eds C. Perna and M. Sibillo. Springer, Dordrecht, pp. 175-182.

[11] Goldstein, S. (1951). On diffusion by discontinuous movements, and on the telegraph equation. Quart. J. Mech. Appl. Math. 4, 129-156.

[12] Gradshteyn, I. S. And Ryzhik, I. M. (2007). Tables of Integrals, Series, and Products, 7th edn. Academic Press, Amsterdam.

[13] KAC, M. (1974). A stochastic model related to the telegrapher's equation. Rochy Mountain J. Math. 4, 497-509.

[14] López, O. and Ratanov, N. (2012). Kac's rescaling for jump-telegraph processes. Statist. Prob. Lett. 82, 1768-1776.

[15] López, O. and Ratanov, N. (2012). Option pricing driven by a telegraph process with random jumps. J. Appl. Prob. 49, 838-849.

[16] Mazza, C. AND Rullière, D. (2004). A link between wave governed random motions and ruin processes. Insurance Math. Econom. 35, 205-222.

[17] Orsingher, E. (1990). Probability law, flow function, maximum distribution of wave-governed random motions and their connections with Kirchoff's laws. Stoch. Process. Appl. 34, 49-66.

[18] Perry, D., Stadje, W. ANd Zacks, S. (2005). A two-sided first-exit problem for a compound Poisson process with a random upper boundary. Methodology Comput. Appl. Prob. 7, 51-62.

[19] Pinsky, M. A. (1991). Lectures on Random Evolutions. World Scientific, River Edge, NJ.

[20] Ratanov, N. (2007). A jump telegraph model for option pricing. Quant. Finance 7, 575-583.

[21] Ratanov, N. (2007). Jump telegraph processes and financial markets with memory. J. Appl. Math. Stoch. Anal. 2007, 19pp.

[22] Ratanov, N. (2010). Option pricing model based on a Markov-modulated diffusion with jumps. Braz. J. Prob. Statist. 24, 413-431. 
[23] Ratanov, N. and Melnikov, A. (2008). On financial markets based on telegraph processes. Stochastics 80, 247-268.

[24] Stadje, W. And Zacks, S. (2004). Telegraph processes with random velocities. J. Appl. Prob. 41, $665-678$.

[25] Van Lieshout, M. N. M. (2006). Maximum likelihood estimation for random sequential adsorption. Adv. Appl. Prob. 38, 889-898.

[26] ZACKS, S. (2004). Generalized integrated telegrapher process and the distribution of related stopping times. J. Appl. Prob. 41, 497-507. 